\title{
Laser characterization of ultrasonic wave propagation in random media
}

\author{
John A. Scales* and Alison E. Malcolm \\ Physical Acoustics Laboratory and Center for Wave Phenomena, Department of Geophysics, Colorado School of Mines, \\ Golden, Colorado 80401
}

(Received 16 December 2002; published 28 April 2003)

\begin{abstract}
Lasers can be used to excite and detect ultrasonic waves in a wide variety of materials. This allows the measurement of absolute particle motion without the mechanical disturbances of contacting transducers. In an ultrasound transmission experiment, the wave field is usually accessible only on the boundaries of a sample. Using optical methods, one can measure the surface wave field, in effect, within the scattering region. Here, we describe noncontacting (laser source and detector) measurements of ultrasonic wave propagation in randomly heterogeneous rock samples. By scanning the surface of the sample, we can directly visualize the complex dynamics of diffraction, multiple scattering, mode conversion, and whispering gallery modes. We will show measurements on rock samples that have similar elastic moduli and intrinsic attenuation, but different grain sizes, and hence, different scattering strengths. The intensity data are well fit by a radiative transfer model, and we use this fact to infer the scattering mean free path.
\end{abstract}

DOI: 10.1103/PhysRevE.67.046618 PACS number(s): 43.35.+d, 42.68.Ay, 74.40.+k, 74.25.Ld

\section{INTRODUCTION}

Rocks are complicated heterogeneous materials with microstructures that scatter acoustic or seismic waves. At the laboratory (ultrasonic) scale, these microstructures are primarily cracks and grain boundaries. The scattering of waves from these boundaries is generally considered noise in geophysical applications, but these scattered wave fields may give insight into how the rock formed, the environment in which it formed, its state of stress, fluid saturation, etc.

Heterogeneous media appear homogeneous when probed with waves whose wavelength is large compared to the scale of heterogeneity. Long-wavelength measurements provide the bulk or average properties of the medium. Traditional acoustic measurement methods using contacting transducers as sources and detectors work well to measure bulk properties, since these properties can be inferred from waves that travel directly from the source to the detector. As the wavelength decreases relative to the heterogeneity, scattering from the microstructure becomes important and the transducers themselves act as scatterers, disturbing the measured wave fields. We avoid these problems by using lasers as both sources and detectors of ultrasonic waves. Using lasers also allows us to collect dense, high-fidelity data sets relatively quickly without having to physically couple transducers to the sample. We can introduce the laser beams into hostile environments (such as vacuum chambers and ovens) via optical windows or fiber, create line or point sources by focusing the source beam, scan the surface wave field by mechanically pointing the detector beam, and focus the detector beam on spots that are much smaller than the smallest contacting transducer. Imagine having an array of tens of thousands of micron sized, massless accelerometers.

The ability to collect dense data sets allows us to visualize the entire wave field as it travels through the rock. By simply

\footnotetext{
*Electronic address: jscales@mines.edu; URL: http://acoustics.mines.edu
}

watching the wave field move as a function of time, it is possible to distinguish effects such as absorption from scattering. To highlight these differences, we perform experiments on samples with different scales of heterogeneity. We have also used three different experimental setups, each to highlight different aspects of wave propagation. Ultimately our goal is to elevate the multiple-scattering speckle for routine use as an important source of information. We start here with the more modest goal of demonstrating the experimental feasibility of our plan and showing how strongly multiply scattered energy can be used to make inferences about the microstructure of rocks.

\section{EXPERIMENTAL SETUP}

In our laboratory, we study ultrasonic wave propagation in heterogeneous media such as rocks and engineered composites. A pulsed Nd:YAG (Yttrium aluminum garnet) laser (1064 nm wavelength, $5 \mathrm{~ns}$ pulses, $0.3 \mathrm{~J} /$ pulse) is used to excite ultrasonic waves via thermoelastic expansion or ablation. The source radiation characteristics are different in the thermoelastic and ablation regimes [1]. In the ablation regime, the explosion of the plasma at the surface creates a reactive force into the sample; in the thermoelastic regime the thermal expansion of the sample creates a dipole radiation pattern. This difference could be significant in experiments which depend strictly on source/detector reciprocity such as coherent backscattering.

These waves are detected using a scanning laser interferometer that measures the absolute particle velocity on the surface of the sample via the Doppler shift. (See Ref. [1], for the general principles of laser vibrometry.) The output of the interferometer is then digitized at 14-bit precision using a digital oscilloscope card attached to a workstation. The lasers and the sample are positioned on an optical bench with vibration isolation. The dominant frequency of the measured waves is $1 \mathrm{MHz}$, a limit imposed by the detector electronics. (At $\mathrm{GHz}$ frequencies, femtosecond lasers have recently been used to visualize ultrasound propagation in crystalline mate- 

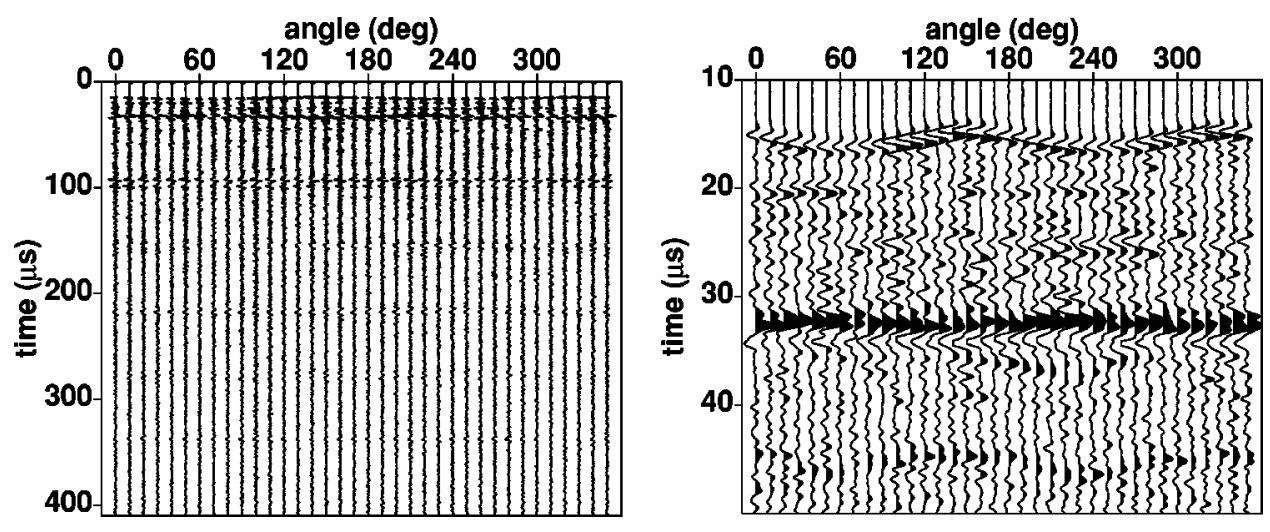

FIG. 1. A rotational scan of the Elberton granite core. The source and detector laser are along a line that crosses the sample at antipodes. The sample is rotated through $360^{\circ}$. On the left is the complete scan with no gain applied. The horizontal events visible are Rayleigh waves. On the right is a zoom showing the first $10-50 \mu \mathrm{s}$ of the data. The first event is a compressional wave propagating directly across the sample. The low frequency event at about $33 \mu$ s consists of two counterrotating Rayleigh waves. The angle-dependent dephasing of these waves is caused by heterogeneities along the path. The horizontal event at about $22 \mu \mathrm{s}$ is a whispering gallery mode that skims the surface of the sample propagating at the compressional wave velocity.

rial [2] by scanning the surface; while in Ref. [3], ultrafast interferometric microscopy is used to characterize laserdriven shock waves.) In the sort of granular rock samples that we use, scattering and attenuation strongly limit the ability to propagate high frequency ultrasound.

We have collected data on three samples representing different scales of heterogeneity: aluminum (homogeneous), Elberton granite (smaller grains), and Llano granite (larger grains). We use three different experimental configurations to highlight different aspects of wave propagation. The first is a "rotational scan," made by shooting across a sample (source and detector beams focused on antipodes, in the middle of a cylindrical sample), which shows the strong surface waves. The second experiment is a "surface scan," in which data are collected on a dense grid on the round top surface of the same cylindrical samples, allowing us to visualize the full wave field. Finally, to extract the scattering mean free path of the strongly scattering Llano sample, we collected data from a rectangular region of a sample large enough to enable us to ignore the strong reflections from the boundaries visible in the surface scan experiment.

\section{SURFACE WAVE TRAINS}

Figure 1 shows an example of a rotational scan in the medium-grained Elberton granite (grain size about $1 \mathrm{~mm}$ ). In this sample, the compressional wave speed $V_{p}$ shows a characteristic sinusoidal dependence on the angle of the sample

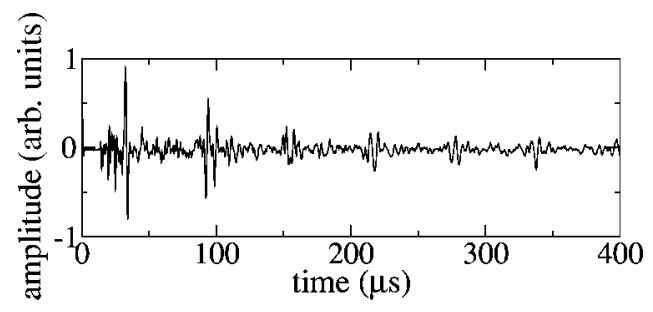

FIG. 2. The average over angle of the time series shown in Fig. 1 . relative to the source/detector line. This body-wave anisotropy is due to the alignment of microfractures in the rock [4]. Only even multiples of the angle appear in a Fourier series of $V_{p}(\theta)$; odd powers are precluded by reciprocity [5]. However, in our configuration, waves that propagate along the surface, such as Rayleigh waves and whispering gallery modes, are unaffected by the anisotropy. The sourcegenerated signal that is incoherent with respect to angle, is largely due to grain scattering. At long times, surface waves dominate the signal since they spread only in two dimensions. If we were to average all these time series over angle, the body waves and the grain-scattered waves would tend to cancel, while the surface waves would be enhanced. This mean, or coherent, time series is shown in Fig. 2. These repetitive surface wave pulses are phase-locked since they originate from the same pulse.

As is well known, a train of phase-locked pulses gives rise to a harmonic comb of frequencies (e.g., Refs. [6,7]). The theory is explained in the Appendix, but the basic idea is that no matter how widely separated in the time domain, the
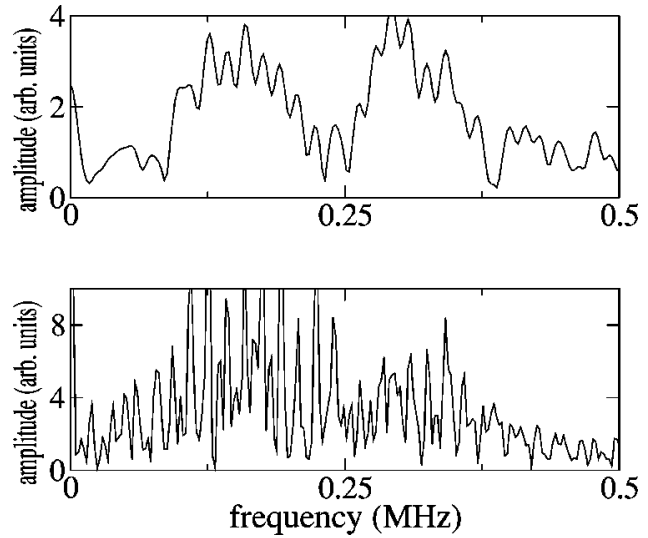

FIG. 3. Power spectra for Fig. 2. In the top part, we tapered around the first two surface wave pulses and then zero padded to the same length as the original data. Below this, we show the power spectrum for the entire time series. 


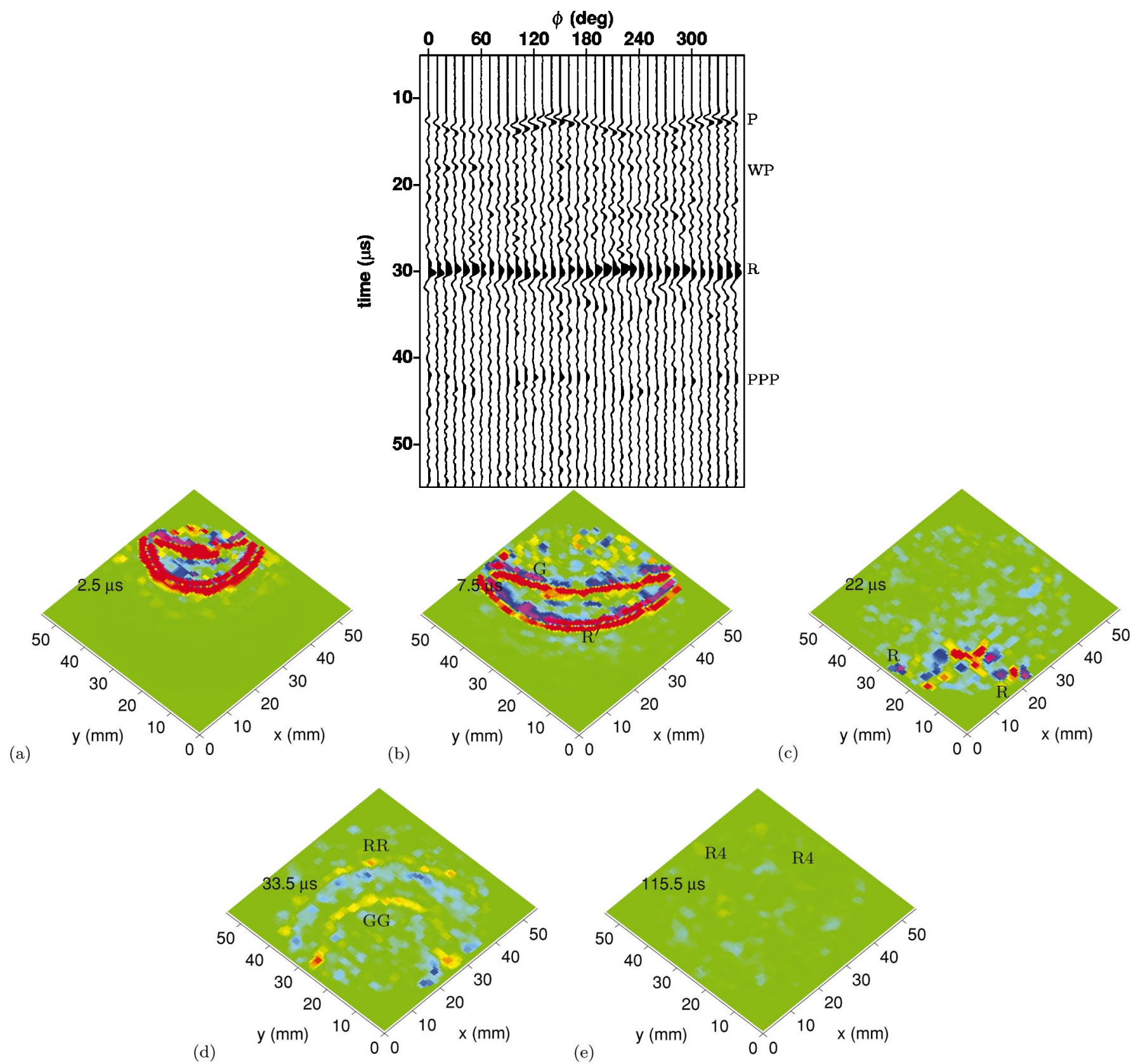

FIG. 4. (Color) Elberton granite: on the top is the early part of the rotation scan of the sample as previously discussed. The full scan shows up to 6 surface wave circumnavigations of the sample. On the bottom are snapshots of the wave field measured on one end of the cylinder. The source laser is focused on a point $7 \mathrm{~mm}$ from the edge of the cylinder. Although the surface wave fields (Rayleigh waves) dominate the figure, both compressional and shear body waves are visible as well as Rayleigh and whispering gallery waves propagating around the edge of the cylinder. In the snapshots, $G$ refers to the so-called ghost wave, which is a reflection of the source from the side of the sample. $R^{\prime}$ is a Rayleigh wave on the end of the sample and $R$ is a Rayleigh wave propagating around the circular edge $(R 4$, for instance, denotes the Rayleigh wave that has gone around the sample four times).

pulses interfere, giving rise to fringes in the power spectrum. These fringes provide a precisely spaced ruler that converges to a Dirac comb as the number of pulses goes to infinity.

Even though we recorded only six pulses, the enhancement of the spectral resolution is striking. To show this, we tapered the coherent signal around the first two pulses and then zero padded to the length of the original time series. The power spectra of the two cases (two pulses versus six) are shown in Fig. 3.
In order to compute the Rayleigh wave speed, we picked the peaks of the power spectrum. The spacing between these peaks corresponds to the rate at which surface waves propagate around the sample. We interpolated (by zero padding) the data in Fig. 2 to a bin size of $305 \mathrm{~Hz}$ to give us a nonzero variance of the peak spacing as a function of frequency. The resulting average peak spacing is $16378 \mathrm{~Hz}$, yielding a surface wave velocity of $2.83 \pm 0.05 \mathrm{~mm} / \mu \mathrm{s}$, for a $55-\mathrm{mm}$ diameter sample. (The uncertainty was conservatively taken 


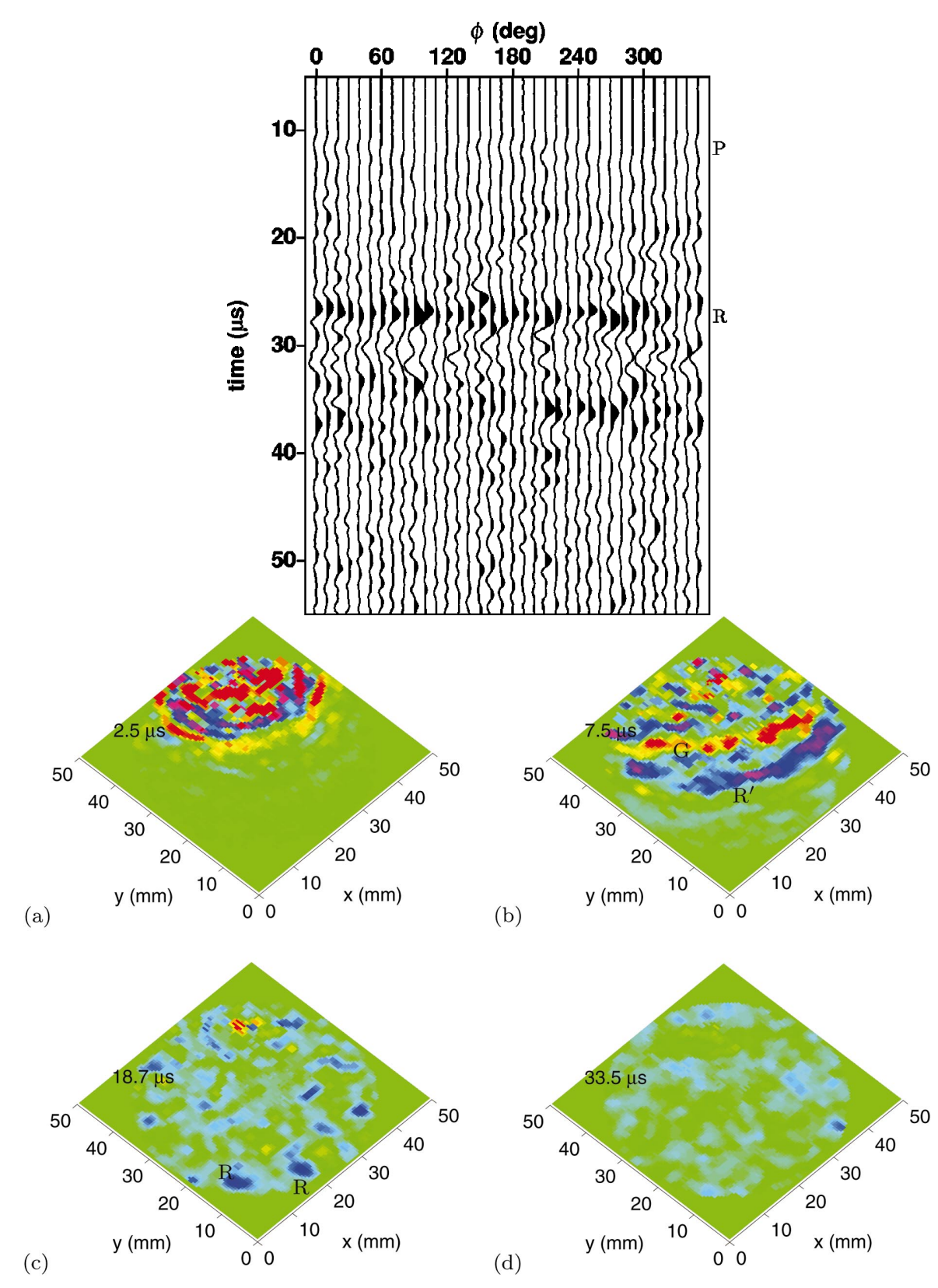

FIG. 5. (Color) Llano granite: in this sample, even though the elastic moduli and intrinsic attenuation are similar to those in the Elberton granite, the scattering is much stronger, since the ratio of wavelength to grain size is close to 1 . This strong scattering manifests itself in the rapid spatial decoherence of the wave field, both in the rotational scan (top) and in the surface wave field snapshots (bottom). After about $30 \mu \mathrm{s}$, the wave field is completely decoherent, consisting entirely of multiple scattering speckle.

to be the $305 \mathrm{~Hz}$ frequency bin size.) By looking at the frequency comb, we can conclude that there is no dispersion (wave speed is independent of frequency) and hence, the microfracturing is not limited to the depth of penetration of the higher surface wave modes.

\section{VARIATION IN SCATTERING STRENGTH}

In the Elberton granite, the ratio of wavelength to grain size is about 3 for surface and shear waves, and 6 for compressional waves. We can see the effects of grain scattering in the coda (i.e., the energy after the ballistic arrivals), but the wave propagation is coherent for hundreds of wavelengths. We have also made measurements in the Llano granite, which has similar elastic moduli and intrinsic attenuation (based on resonance measurements), but much stronger scattering. The ratio of the wavelength to the grain size in this sample is close to 1 .

In order to compare in detail the scattering of ultrasonic waves for these two samples, we performed surface scans on the ends of cylindrical samples. The source laser was focused to a point on one end of the cylinders $7 \mathrm{~mm}$ from the edge. The ends of the cylinders were scanned at a density of 9 points per square millimeter. Both granite cylinders are approximately $100 \mathrm{~mm}$ long. The Elberton granite sample is 55 


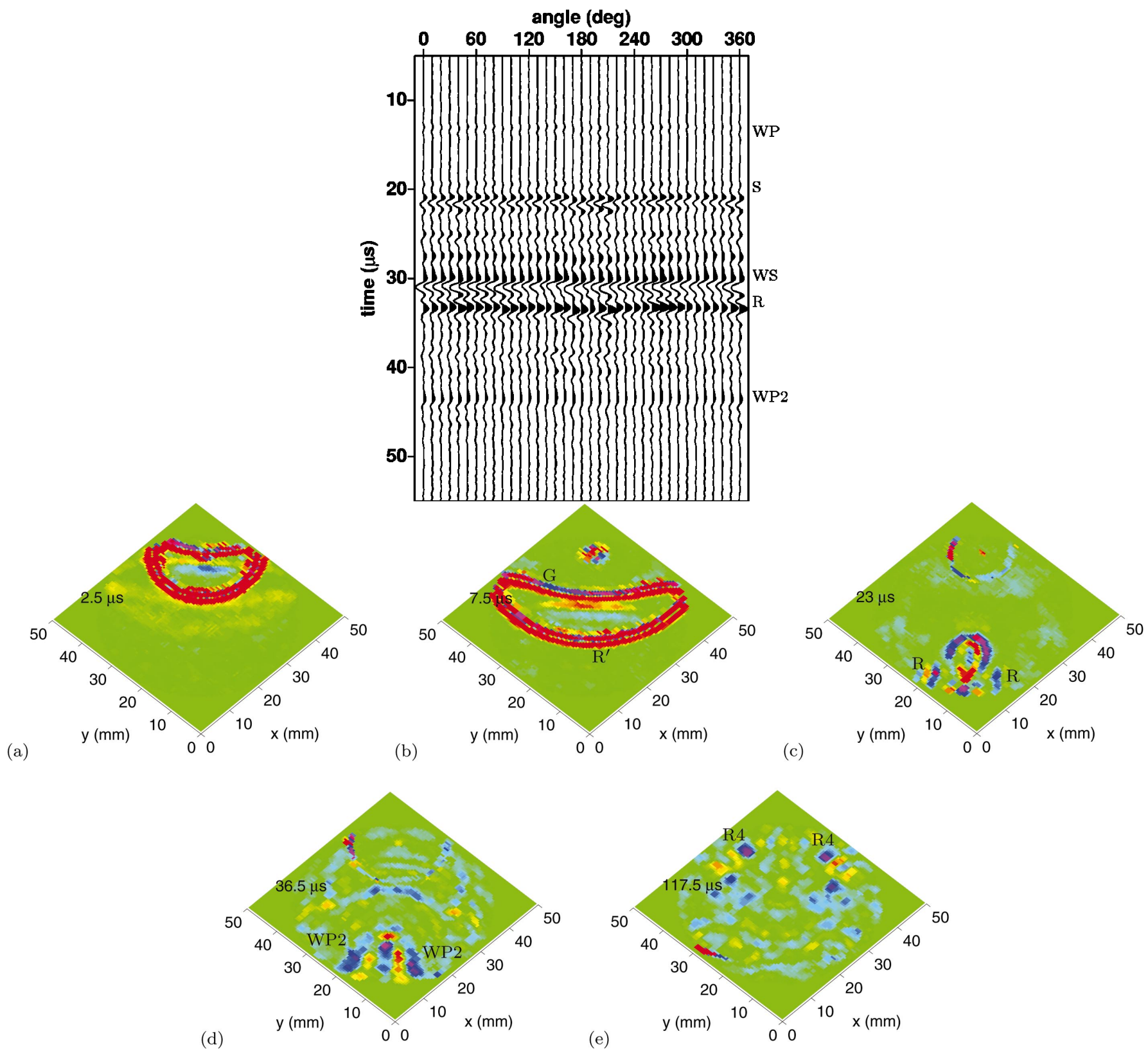

FIG. 6. (Color) Many more modes are visible in the aluminum sample, including whispering gallery modes associated with both shear waves (WS) and compressional waves (WP). In addition, due to the conductivity of the sample, there is an ablation generated sound wave in air visible as a slow outgoing circular wave front in the snapshots. For the rock samples, the source laser was tuned to the thermoelastic regime.

$\mathrm{mm}$ in diameter and the Llano sample is $50 \mathrm{~mm}$ in diameter. Having such spatially dense sampling of the wave field allows for a variety of spatial-Fourier-domain filtering procedures. For example, in Ref. [8], it is shown how to separate the direct from the scattered field in media where surface scatterers are embedded in a background matrix. Here, we limit the discussion to the estimation of the scattering mean free path.

In this experiment, we are almost measuring the Green's function. When tuned to the thermoelastic regime, the radiation pattern of the source laser is not quite reciprocal to the detector [1]. (In the multiple scattering coda, the Green's function can be synthesized from the two-point correlation function of the speckle [9]. This relies on the equipartitioning of the energy among the modes, which only occurs beyond the mean free time.)

Figures 4 and 5 show the rotational scans as above, next to snapshots of the scanned wave field (see Ref. [13]). For comparison, we show in Fig. 6 a surface scan made in aluminum. Given that the intrinsic attenuation of the Llano sample is about the same as that of the Elberton, it is visually apparent that the scattering mean free path $\ell_{s}$ is much shorter in Llano than in Elberton. To quantify this, we look at fitting the data with a radiative transfer model. 


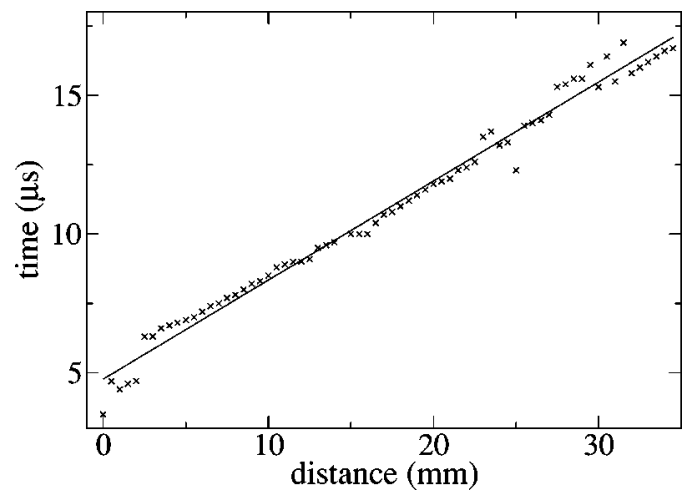

FIG. 7. The group velocity is estimated by performing a linear regression on the peaks of the coherent intensity as a function of source/detector offset.

\section{RADIATIVE TRANSFER}

An analytic solution for a two-dimensional radiative transfer (RT) equation, as given in Ref. [10] is

$$
\begin{aligned}
I(r, t)= & e^{-c t\left(1 / \ell_{a}+1 / \ell_{s}\right)}\left(\frac{\delta(c t-r)}{2 \pi r}+\frac{1}{2 \pi \ell_{s} \sqrt{1-\frac{r^{2}}{c^{2} t^{2}}}}\right. \\
& \left.\times \exp \frac{c t}{\ell_{s}} \sqrt{1-\frac{r^{2}}{c^{2} t^{2}} H(c t-r)}\right),
\end{aligned}
$$

where $c$ is the group or energy velocity, $r$ is the distance from the source, $H$ is the Heaviside function, and $I(r, t)$ is the total intensity. (This model assumes no mode conversion or localization and that the scattering is isotropic.) At "early" times, this corresponds to the ballistic propagation of energy. At "late" times, RT is equivalent to diffusion. Early and late are, of course, relative to the strength of the scattering and attenuation. Our cylindrical surface scans were not ideal for this analysis due to the influence of the boundary. In order to eliminate the effects of the boundary, we made a surface scan on a long block of Llano granite $\left(40 \times 80 \mathrm{~mm}^{2}\right.$ cross section and over $1 \mathrm{~m}$ in length). We focused the source laser onto a 21-mm line using a cylindrical lens and scanned the surface wave field in a region $40 \times 40 \mathrm{~mm}^{2}$ in size. Using a line source gave largely one-dimensional propagation and allowed for ensemble averaging normal to the propagation direction. In order to fit these data to RT, we must first estimate the group or energy velocity. This we do by performing a regression on the peak intensity as a function of source/ detector offset. This regression is shown in Fig. 7.

In the Llano granite, because of the strong scattering, our data do not constrain the absorption $\ell_{a}$ though they do give information about the scattering. Extracting $\ell_{s}$ from the radiative transfer equation is difficult however, because at late times the dependence of RT on $\ell_{s}$ becomes algebraic rather than exponential. Thus, it is important to average many real-

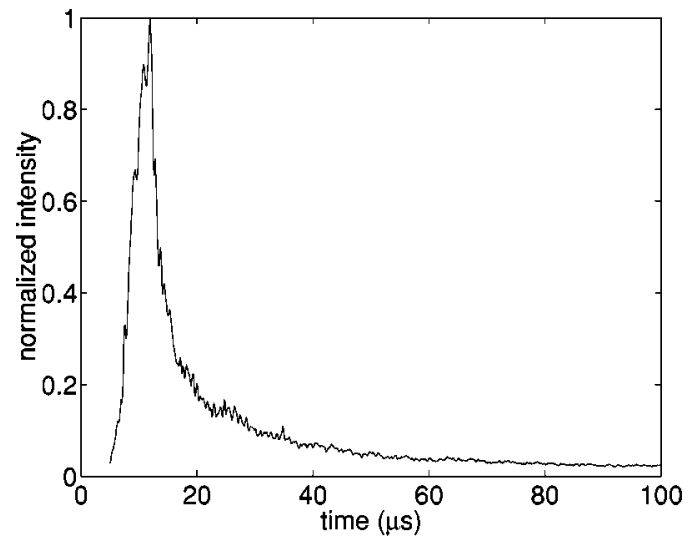

FIG. 8. Once the coherent pulse has passed the detector (i.e., after about $15 \mu \mathrm{s}$ ), the total intensity is essentially independent of distance within the scan region. The intensity, averaged over source/ detector distance, is shown here. The broad peak here is the result of averaging the ballistic energy over different distances.

izations to reduce the spread of the data enough to constrain $\ell_{s}$. To do this, we note that at large times (i.e., well after the ballistic peak), RT is independent of the distance $r$, allowing us to average over $r$ as well as over our ensemble of different lines of detector positions. The ensemble average intensity is shown in Fig. 8. We then fit the result to RT, as shown in Fig. 9 , giving us a mean free path of about $8 \pm 2 \mathrm{~mm}$.

\section{CONCLUSIONS}

Laser ultrasonics provides an ideal tool for studying the complex dynamics of wave propagation in random media. It is possible to scan the surface of objects hundreds of wavelengths or more in extent at a sufficient density (many grid points per wavelength) to allow for the visualization of the surface wave field. Using a pulsed IR laser as a source allows us to create sources that are focused on points, lines or other distributed shapes, while using a scanning laser interferometer as a detector is analogous to having an array of tens of thousands of massless accelerometers. In addition, the interferometer provides an absolute measure of particle motion. Our goal is to use these noncontacting methods to measure

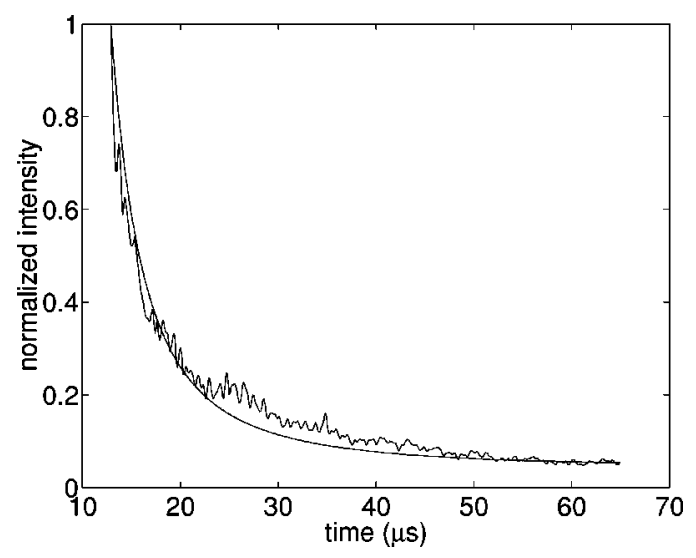

FIG. 9. A radiative transfer fit to the late-time intensity for mean free scattering path of $8 \mathrm{~mm}$. Analysis of the optimization problem for $\ell_{s}$ gives an uncertainty of about $\pm 2 \mathrm{~mm}$. 
and exploit the multiple scattering speckle in random media such as rock. Here we have shown an analysis of measurements made on three samples of varying degrees of heterogeneity. For the most heterogeneous sample, a coarsegrained granite, the scattering is so strong that the wave field rapidly decoheres into speckle. In this sample, we are able to infer the scattering mean free path by fitting a radiative transfer model to the ensemble averaged intensities. Thus, we can exploit the multiple scattering to characterize a microscopic feature of the rock sample that would be difficult to measure otherwise.

\section{ACKNOWLEDGMENTS}

We acknowledge many useful discussions with our CSM colleagues Roel Snieder, Kasper van Wijk, Alex Grêt, Matt Haney, Huub Douma, and Carlos Pacheco and with Bart van Tiggelen of the Université Joseph Fourier in Grenoble. This work was partially supported by the National Science Foundation (Grant No. EAR-0111804) and the U.S. Army Research Office (DAAG55-98-1-0070). A.E.M. was partially supported by TotalFinaElf.

\section{APPENDIX: PHASE-LOCKED PULSE TRAINS}

Consider a Gaussian pulse $p(t)=e^{-(t-\Delta t)^{2} / 2 \sigma^{2}}$. The time shift introduces a phase shift in the Fourier transform: $p(\omega)=\sigma e^{-i \omega \Delta t} e^{-\sigma^{2} \omega^{2} / 2}$. Hence, the power spectrum for two identical pulses separated by $\Delta t$ will contain an interference term. The envelope will still be the Gaussian $e^{-\sigma^{2} \omega^{2}}$, but there will be modulation with peaks of full-width at half- maximum (FWHM) $\pi / \Delta t$ and separation $1 / \Delta t$. For $n$ such pulses, the power spectrum is a geometric series, the summation of which is

$$
P_{n}(\omega)=2 \sigma^{2} e^{-\sigma^{2} \omega^{2}}\left(\frac{1-\cos (\omega n \Delta t)}{1-\cos (\omega \Delta t)}\right)
$$

In the $n$-pulse case, the spacing between the peaks of the power spectrum is still $1 / \Delta t$ but the FWHM is $2 \pi / n \Delta t$. If $\Delta t$ is constant, then for large $n$, the pulse superposition provides a precise ruler in the frequency domain, allowing pulse trains to achieve bandwidth far below that of a single pulse (see Ref. [11], for a historical account of the development of the pulsed laser comb). In the limit that the number of pulses goes to infinity, Eq. (A1) converges to a comb of Dirac $\delta$ functions with a Gaussian envelope. In a way this is not surprising, since standing waves (i.e., normal modes), which have line spectra, can be thought of as a superposition of a large number of traveling waves. In fact, in our experiment the comb is nothing but the fundamental surface wave mode plus all the higher overtones.

For our cylindrical sample, the number of pulses that can be used depends on the $Q$ of the sample. Of course, once the frequency of the fundamental surface wave mode is known, we could perform a resonance experiment by exciting the sample near this frequency and detecting the signal with a lock-in amplifier as in resonance ultrasound spectroscopy [12]. Still, the frequency comb gives us direct access to any frequency dependence (dispersion) of the surface wave speed, and hence, the depth of penetration of the microfracturing.
[1] C. Scruby and L. Drain, Laser Ultrasonics: Techniques and Applications (Adam Hilger, London, 1990).

[2] Y. Sugawara et al., Phys. Rev. Lett. 88, 185504 (2002).

[3] K.T. Gahagan et al., J. Appl. Phys. 92, 3679 (2002).

[4] P. Douglass and B. Voight, Geotechnique 19, 376 (1969).

[5] M. Smith and F. Dahlen, J. Geophys. Res. 78, 3321 (1973).

[6] R. Teets, J. Eckstein, and T. Hänsch, Phys. Rev. Lett. 38, 760 (1977).

[7] M. Bellini, A. Bartoli, and T. Hänsch, Opt. Lett. 22, 540 (1997).

[8] X. Campman and K. van Wijk (unpublished).
[9] A. Malcolm, J. Scales, and B. van Tiggelen (unpublished).

[10] J.C. Paasschens, Phys. Rev. E 56, 1135 (1997).

[11] T. Udem and A. Fergusen, in Laser Physics at the Limits, edited by H. Figger, D. Meschede, and C. Zimmermann (Springer-Verlag, Berlin, 2002).

[12] B. Zadler, J. Le Rousseau, J. Scales, and M. Smith (unpublished).

[13] Mpeg movies of these data are available on our web site at http://acoustics.mines.edu. These movies are very useful for understanding the complex dynamics of wave propagation, scattering, caustic generation, etc. 